\title{
DIFFUSE PROGRESSIVE INTERSTITIAL FIBROSIS OF THE LUNGS IN CHILDHOOD (HAMMAN-RICH SYNDROME)
}

\author{
BY \\ H. B. HILTON and JOHN RENDLE-SHORT \\ From the Sheffield Children's Hospital and the Department of Child Health, University of Sheffield
}

(RECEIVED FOR PUBLICATION JUNE 9, 1960)

Hamman and Rich (1935) briefly described four cases of bilateral diffuse interstitial fibrosis of the lungs of unknown aetiology. In 1944, they described this condition in detail under the title 'Acute diffuse interstitial fibrosis of the lungs' (Hamman and Rich, 1944).

Since then the condition has become known as the 'Hanman-Rich syndrome' but is now perhaps more correctly referred to as 'Diffuse progressive interstitial fibrosis of the lungs' (Beams and Harmos, 1949).

Grant, Hillis and Davidson (1956) collected 36 cases from the literature and added three of their own. Rubin and Lubliner (1957) recognized 39 recorded cases and added 15 of their own. Since then sporadic reports of cases have appeared, and Scadding (1960) added 26 cases to the literature. The majority of reported cases have been in adults; in childhood the disease is rare, only six cases having been previously recorded.

The first report of the condition occurring in a child was by Bradley (1956). He described the clinical and autopsy findings in a 9-year-old girl. Since that time the following cases have been reported: Baar and Braid (1957) described the case of a 3-year-old boy; Feinerman and Harris (1957) of a 7-month-old girl and an 8-year-old boy; Diamond (1958) of a 4-year-old Negro boy who, in addition, had stenosis of the right pulmonary artery and complete occlusion of the right pulmonary vein, and Mann (1959) an 8-month-old girl.

Wilson and Mikity (1960) have recently reported a syndrome resembling the Hamman-Rich syndrome which occurs in premature babies. They gave details of five infants, two of whom had pulmonary signs and symptoms suggestive of the HammanRich syndrome. It is not certain, however, that these should be regarded as proved cases.

Case 1: J.L.

John was the second child of healthy parents. He was born after a normal pregnancy and labour (birth weight $7 \mathrm{lb} .4 \mathrm{oz}$.). He developed normally for the first three months of life and reached a weight of $11 \mathrm{lb} .10 \mathrm{oz}$. He then developed, over the course of a week, a slight fever, cough and 'stuffy' nose.

On admission to the Sheffield Children's Hospital, under the care of Professor Illingworth, he was slightly cyanosed. His temperature was $100^{\circ} \mathrm{F}$., his heart rate 180 per minute and his respiratory rate 30 per minute. A grade $3 / 6$ systolic murmur was audible over the precordium, and both liver and spleen were palpable two fingers' breath below the costal margin. Investigations showed nothing abnormal apart from the chest radiograph, which revealed bronchopneumonic changes throughout both lung fields. He was treated with oxygen, penicillin and digoxin and seemed to improve. His liver became less easily palpable and the systolic murmur disappeared.

$\mathrm{He}$ was discharged home, but four days later was readmitted with marked cyanosis which disappeared when he was placed in an oxygen tent. The systolic murmur had returned and he was dyspnoeic, but had no fever. The liver was not so large, but the chest radiograph showed the same changes as previously.

During the next three months the child's condition steadily deteriorated. He became a blue-grey colour when not receiving oxygen, lost weight and his haemoglobin level rose from $12 \cdot 1 \mathrm{~g}$. \% on his first admission to $14.4 \mathrm{~g}$. \% just before death. It was thought that he probably had a congenital heart lesion with cardiac failure. The electrocardiogram showed evidence of right ventricular diastolic overloading, but a cine-angiocardiogram was normal. Repeated blood cultures were negative. He was treated with tetracycline on four occasions, penicillin twice, chloramphenicol once, and was also given digoxin, triamcinolone and nystatin for persistent oral thrush. No therapy except oxygen appeared to help him. All investigations remained negative, but the chest radiograph showed an increasing haziness of the whole lung field with an almost ground-glass appearance (Fig. 1). He died at the age of $6 \frac{1}{2}$ months.

Necropsy. The body was that of an extremely wasted male infant weighing $3,097 \mathrm{~g}$. Scattered petechial haemorrhages were present over the face, abdomen and ankles, and fading petechial haemorrhages were present in both groins. 
ResPiRATORY TRACT. Lesions of thrush were present in the mouth and pharynx. The trachea and main bronchi appeared to be normal.

Both lungs were bulky and voluminous and filled the thoracic cavity. There were depressions on the posterior surface of both lungs which corresponded with the posterior aspect of the ribs. These depressions had apparently been produced by pressure of the lungs against the thoracic cage. All lobes of both lungs were firm and gave the appearance of consolidation, but there was no congestion or oedema. The smaller bronchi and bronchioles appeared normal and contained no exudate. The pleural surface of both lungs showed some dilatation of vessels. The cut surface of the lungs had a mottled appearance, consisting of reddish and whitish areas, and also showed a diffuse lace-like pattern of firm whitish fibrous tissue (Fig. 2). The tracheo-bronchial and some of the mediastinal lymph nodes were slightly enlarged, a little congested and soft, but there was no evidence of caseation or calcification.

Cardiovascular System. There was moderate distension of the jugular veins and a distended azygos vein was present. The heart appeared to be normally developed. There was no congenital abnormality and no evidence of right ventricular hypertrophy.

The liver was slightly enlarged and congested.

The brain showed engorgement of the cerebral veins.

All other organs appeared normal.

Histological Findings. The following appearances were found in approximately equal severity in all lobes of both lungs: there was a great increase in the thickness of the alveolar wall, apparently due to a combination of oedema and increase in argentaffine fibres. Large mononuclear cells and a few lymphocytes were present in the interstitial tissue but polymorphonuclear leucocytes and eosinophil leucocytes were scanty. There was marked vascularity of the interstitial tissue. Many alveoli were dilated and the alveolar epithelium was

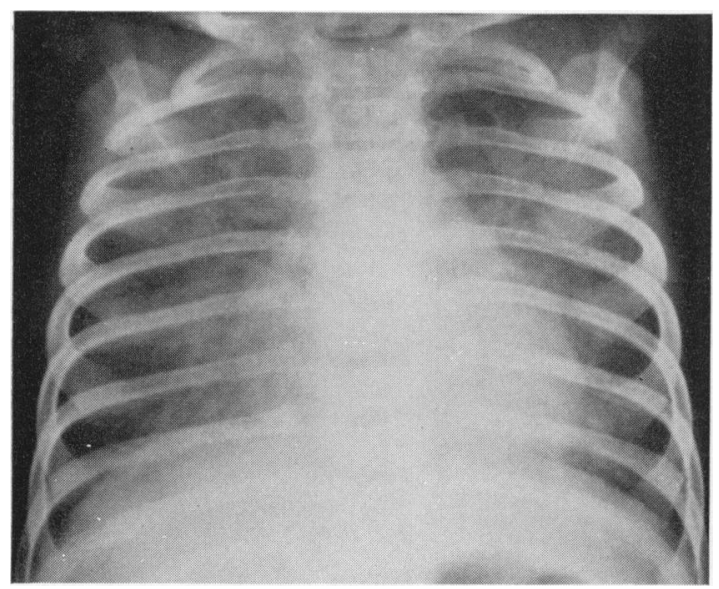

Fig. 1.-Case 1, chest radiograph showing 'ground glass' appearance of lung fields.

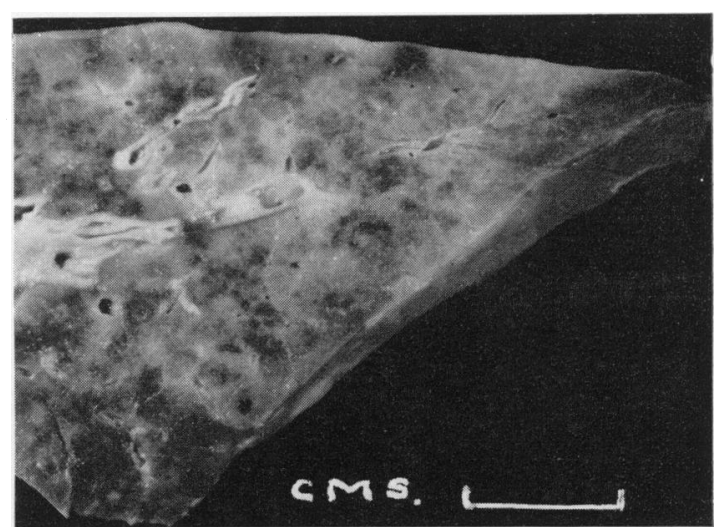

FIG. 2.-Case 1, cut surface of lung showing mottled appearance.

replaced by increased amounts of connective tissue which produced a 'pseudo-hyaline membrane' appearance. Many other alveoli were not dilated but were small and lined by cuboidal epithelium. Alveoli, alveolar ducts, bronchioli and bronchi were either empty or contained a few mononuclear cells which appeared to be swollen alveolar epithelial cells which had become separated from the alveolar wall. No evidence of fungal infection was seen.

\section{Case 2: A.R.}

Angela was the third child of healthy parents (birth weight $6 \mathrm{lb}$.). She had been perfectly well until she was vaccinated against smallpox at the age of 14 weeks. She had a strong reaction to the vaccination and the scab had still not separated five weeks later. Three days after the vaccination she began to vomit and had a bout of diarrhoea which lasted five days. Four days after the vaccination she started to cough and this persisted. At that time she showed no evidence of cyanosis. During the next four weeks the cough became worse. The child refused feeds, vomited occasionally and then became cyanosed.

When seen by Dr. L. A. Hawkins at the Doncaster Royal Infirmary, Angela was 18 weeks old. She was a greyish blue colour with a respiration rate of $60 \mathrm{per}$ minute. There was no evidence of respiratory obstruction. Widespread crepitations were audible. The pulse rate was uncountable. No abnormality was noted in the heart. The temperature was $101^{\circ} \mathrm{F}$.

On admission she was put into an oxygen tent and her colour rapidly became normal. This rapid improvement in response to oxygen continued until just before her death and was a notable feature of the case. Apart from oxygen, Angela was treated with penicillin (two courses), chloramphenicol, tetracycline, chlortetracycline and streptomycin but without any improvement. Radiological examination showed a widespread, mottled opacity of both lung fields, so much so that almost no normal lung translucency could be seen. It was difficult to distinguish the heart from the lungs and no abnormality of the heart was observed. 
Angela's condition deteriorated and she died at the age of 7 months, 10 weeks after the onset of her illness.

Necropsy. The lungs were voluminous and on the posterior aspects, slight markings were present which corresponded to the posterior part of the ribs. The lungs were firm and on cut section showed a fleshy appearance with a variegated pattern of pale and reddish areas. No definite consolidation was present and no frank pus was seen.

Histological Findings. All lobes of both lungs showed a similar appearance. There was gross thickening of the alveolar walls which appeared to be due to a combination of increased connective tissue, oedema and cellular infiltrate of mononuclear cells and occasional eosinophil leucocytes. Dilated capillaries were present in the interstitial tissue. The majority of alveoli were lined by cuboidal epithelium; other alveoli were distended. The smaller bronchi, bronchioles and alveoli were either empty or contained swollen mononuclear cells which appeared to have been derived from the alveolar epithelium. The heart showed no gross abnormality and the other organs were normal.

\section{Case 3: L.S.}

Linda was well until the age of 13 months when she developed a respiratory tract infection with otitis media and a cough. The otitis improved after two weeks, but the cough became progressively worse and the respiratory rate became rapid. She was admitted to the Sheffield Children's Hospital under the care of Professor Illingworth.

On examination she was a pale, coughing baby with a tinge of cyanosis. The temperature was $100^{\circ} \mathrm{F}$., and the respiratory rate about 80 per minute. On auscultation of her chest, crepitations were heard all over both lung fields. A radiograph showed emphysematous lung fields with patchy consolidation and one possible cavity in the right mid-zone. The pulmonary artery was prominent.

Linda was put into an oxygen tent and treated with tetracycline. Her haemoglobin was $12.9 \mathrm{~g}$. $\%$ and her total leucocyte count $21,000,68 \%$ of which were neutrophils. Gastric washings on admission showed no pathogenic organisms.

There was a slight initial improvement, but after the first two weeks she began to deteriorate. She became very cyanosed when out of oxygen, but was comparatively cheerful in the oxygen tent. The pulmonary crepitations were always audible, although her cough became less troublesome. Serial radiographs showed increasing emphysema with areas of consolidation uniformly distributed throughout both lung fields (Fig. 3). No abscess cavities were seen. Her heart enlarged progressively, especially the right ventricle, and the pulmonary artery became very prominent. Her haemoglobin rose, until just before death it was $16 \cdot 7 \mathrm{~g}$. $\%$. Staphylococcus aureus coagulase positive was grown from cough swabs on eight occasions in specimens taken at aproxpimately monthly intervals.
Linda was treated with courses of penicillin, chlortetracycline, oxytetracycline, sulphadimidine, chloramphenicol and erythromycin. She was also given cortisone for two months and, when she was in heart failure, digoxin. No treatment, apart from oxygen, made any difference to her general condition or altered the persistent growth of Staphylococcus aureus in her sputum, even though in vitro studies showed that the organisms remained fully sensitive to chloramphenicol and erythromycin throughout.

Linda developed progressive cor pulmonale as shown by chest radiographs and electrocardiograms, and she died in heart failure eight months after the initial appearance of her symptoms.

Necropsy. The body was that of a wasted female child, the sternum was prominent and the anteroposterior diameter of the chest was increased.

Respiratory Tract. The larynx, pharynx, trachea and larger bronchi appeared normal.

The lungs were slightly more bulky than normal and the pleural surface showed irregularity due to the presence of small sub-pleural cysts which varied in size from $1 \mathrm{~mm}$. to $1 \mathrm{~cm}$. in diameter. The pleural surface showed mottled areas of red congested tissue and white depressed areas due to fibrous tissue. On cut section the mottled appearance was seen to be present throughout all lobes of both lungs.

Cardiovascular System. The heart was increased in size due to right ventricular hypertrophy. There was no evidence of congenital heart disease. The pulmonary artery appeared slightly increased in thickness. There was distension of the jugular veins.

The liver was enlarged and congested. The thymus showed marked involution. All other organs were normal.

Histological Findings. Throughout all lobes of both lungs, there was an increase in thickness of the alveolar

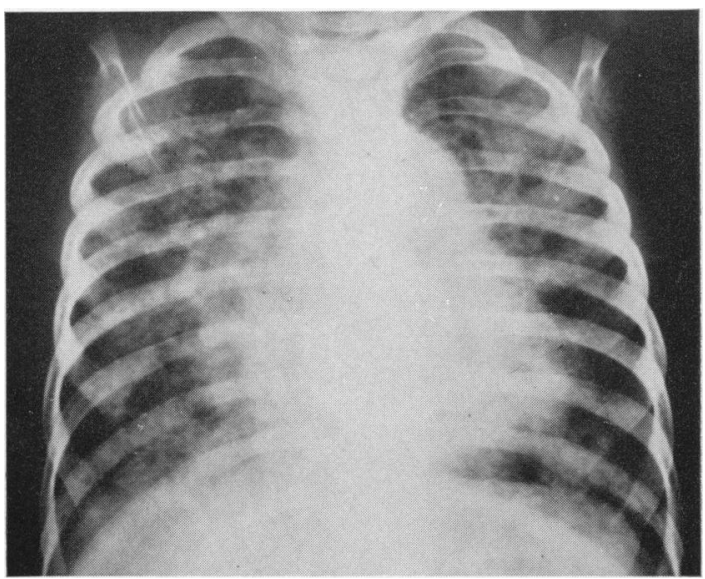

FIG. 3.-Case 3, chest radiograph showing areas of consolidation, enlargement of the heart and prominent pulmonary artery. 
walls. This was due to increased amounts of connective tissue and collections of cells which included macrophages, fibroblasts, plasma cells and some eosinophils. Many alveoli appeared small or normal in size and were lined by cuboidal epithelium (Fig. 4). Other alveoli were dilated and cysts were present which were lined in some areas by a thin layer of fibrous tissue, and in other areas by ciliated columnar epithelium.

The heart showed slight hypertrophy of muscle fibres in the right ventricle. There was no evidence of myocarditis.

The pulmonary artery showed some sub-intimal thickening. The thymus showed gross involution. The liver showed congestion and fatty degeneration. The spleen was congested but was otherwise normal.

\section{Discussion}

An analysis of the six previously recorded childhood cases (excluding the five premature babies) in conjunction with our own three cases, enables a composite picture of the syndrome to be given. The sex incidence is approximately equal (four boys and five girls). The ages at onset were: 3 months (two cases), 4 months (two cases), 5 months (two cases), 13 months, 2 years 5 months and 6 years 9 months. Five cases survived six months or less, while three cases survived between eight months and three and a half years. One 8-year-old boy was still alive at the time his case history was published one year after the onset of the disease.

Five cases began with an upper respiratory tract infection. Two cases began with fever and respiratory tract symptoms. One case apparently followed whooping cough and one case followed vaccination against smallpox. The usual story was

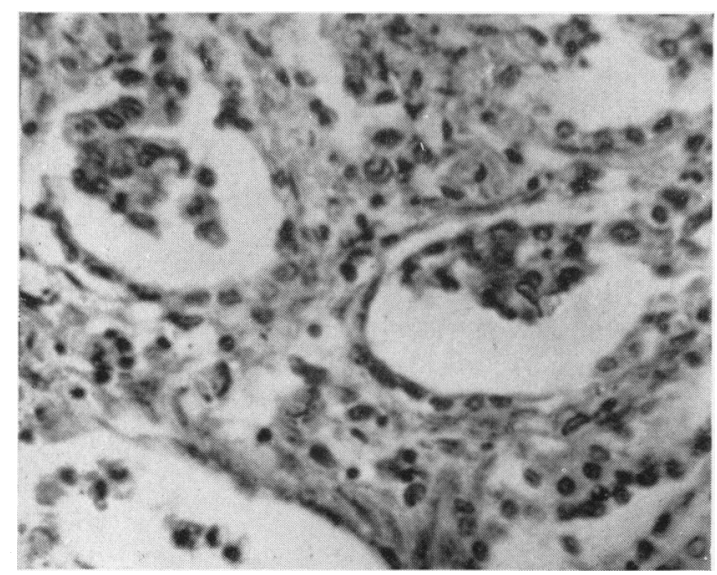

FIG. 4.-Case 3, lung section showing broadening of interalveolar interstitial tissue and cuboidal epithelium lining the alveoli. (H. and E. $\times$ 295.) of cough, dyspnoea and cyanosis persisting after an infection had apparently abated. The cyanosis was relieved by oxygen. Several authors remarked on the remarkable absence of signs in the chest, but some of the children were seen months after the commencement of the disease. On the other hand a few cases had persistent crepitations throughout the illness.

Six out of seven cases died in heart failure. In the child who was still alive, the cardiac second sound was reported as being very loud and split. In one case report the heart was not mentioned.

In our first child the presence of a systolic murmur which was shown to be presystolic on a phonocardiogram, coupled with dilatation of the pulmonary artery on a radiograph, right ventricular overloading on the electrocardiogram and clinical evidence of right-sided heart failure, suggested the diagnosis of a congenital heart lesion. An angiocardiogram, however, was normal. A similar case was reported by Bradley (1956); the child had a loud systolic and later diastolic murmur and cardiac catheterization was performed. A general increase of pressures in the right side of the heart was found (figures not given), but no other abnormality was discovered. At autopsy no congenital heart lesion was found.

The radiological appearance of the nine cases varied. Two cases showed patchy consolidation and emphysema, five cases showed diffuse infiltration, and two cases showed a ground glass haziness of the lung fields. The abnormal appearance was often more marked on one side than the other.

In our third case the diagnosis of staphylococcal pneumonia either with or without fibrocystic disease of the pancreas was seriously considered. This was because of the radiological appearance of patchy consolidation and possible pneumatoceles, and also because of the constant finding of haemolytic staphylococci in the sputum.

Diffuse progressive interstitial fibrosis of the lungs is a clinical syndrome which should be considered in any child with persistent dyspnoea, tachypnoea, cyanosis improved by oxygen, and heart failure. The most important differential diagnosis is congenital heart disease.

Aetiology. Various possibilities have been suggested as aetiological factors in cases of 'idiopathic' diffuse interstitial pulmonary fibrosis. These include a reaction to some form of chemical irritant, the result of a viral infection or infection with pleuropneumonia-like organisms or an incompletely resolved pneumonia. A relationship with the collagen disease has also been suggested, and cases 
of rheumatoid arthritis with progressive pulmonary fibrosis have been recorded in adults (Rubin and Lubliner, 1957).

The concept that auto immunity may be concerned has received some support from Read (1958a and 1958b) who prepared, in rabbits, an anti-rat lung serum which, when administered to rats, resulted in interstitial fibrosis of the rat lung.

Pathology. The naked eye appearances of the lung in all our three cases showed a similar appearance. The lungs were voluminous and increased in bulk. They were uniformly firm in consistence and the cut surface showed a variegated pattern of greyish white and pale reddish areas. In two cases there were prominent dilated blood vessels present on the pleural surface. In one case, the presence of small cysts was noted.

Histological Findings. The histopathological findings in our three cases fulfilled the criteria described by Hamman and Rich. The main pathological feature was broadening of the interstitial tissue of interalveolar septa. This pathological change involved all lobes of both lungs. The broadening of interstitial tissue was due to oedema, increased numbers of argentaffine and collagenous fibres and cellular infiltration. The cellular infiltrate comprised large mononuclear cells, lymphocytes, plasma cells and eosinophil leucocytes in varying proportions in the three cases.

In Case 3 of our series frequent isolation of haemolytic Staphylococcus aureus from the sputum, together with the cystic changes in the lungs, was suggestive of staphylococcal pneumonia. In organizing pneumonia, however, changes are usually lobar or lobular and not diffuse and even if both lungs were involved uniformly, the alveoli themselves are filled with organized exudate. In this case there was no organized alveolar exudate, no evidence of suppuration and the walls of the cyst were lined by a thin layer of fibrous tissue and in some parts respiratory epithelium. The cysts were probably produced by dilatation of bronchioles and there was no evidence of a surrounding inflammatory reaction.

\section{Summary}

Three cases of the Hamman-Rich syndrome in childhood are described. The ages of the children at death were $6 \frac{1}{2}, 7$ and 23 months.

These cases were manifested clinically by dyspnoea, tachypnoea and cyanosis. The respiratory symptoms became steadily more marked and two of the children died with right-sided cardiac failure.

The pathological findings in the lungs were similar in all three cases, the main macroscopic appearances consisting of increase in bulk of the lungs with evidence of fibrosis, and the main histological feature being broadening of the interalveolar interstitial tissue.

No treatment was of any avail apart from oxygen.

The Hamman-Rich syndrome should be considered in any child who has persistent cough, dyspnoea, tachypnoea, cyanosis improved by oxygen, and heart failure.

We would like to thank Professor R. S. Illingworth, under whom two of these cases were admitted, and Dr. J. L. Emery for kindly criticizing this paper. Dr. L. A. Hawkins kindly supplied us with the clinical details of Case 2.

\section{RefERENCES}

Baar, H. S. and Braid, F. (1957). Diffuse progressive interstitial fibrosis of the lungs in childhood. Arch. Dis. Childh., 32, 199.

Beams, A. J. and Harmos, O. (1949). Diffuse progressive interstitial fibrosis of the lungs. Amer. J. Med., 7, 425.

Bradley, C. A. (1956). Diffuse interstitial fibrosis of the lungs in children. J. Pediat., 48, 442.

Diamond, I. (1958). The Hamman-Rich syndrome in childhood. Report of a case with unilateral pulmonary arterial and venous stenosis and atriovenous occlusion. Pediatrics, 22, 279.

Feinerman, B. and Harris, L. E. (1957). Unusual interstitial pneumonitis. Report of two cases occurring in children. Proc. Mayo Clin., 32, 637.

Grant, I. W. B., Hillis, B. R. and Davidson, J. (1956). Diffuse interstitial fibrosis of the lungs (Hamman-Rich syndrome). Amer. Rev. Tuberc., 74, 485.

Hamman, L. and Rich, A. R. (1935). Fulminating diffuse interstitial fibrosis of the lungs. Trans. Amer. clin. Climatol. Ass., $51,154$. (1944). Acute diffuse interstitial fibrosis of the lungs. Bull. Johns Hopk. Hosp., 74, 177.

Mann, T. P. (1959). Diffuse progressive interstitial fibrosis of lungs in infancy. Proc. roy. Soc. Med., 52, 638.

Read, J. (1958a). The pathogenesis of the Hamman-Rich syndrome. A review from the standpoint of possible allergic etiology. Amer. Rev. Tuberc., 78, 353.

(1958b). The pathological changes produced by anti-lung serum. J. Path. Bact., 76, 403.

Rubin, E. H. and Lubliner, R. (1957). The Hamman-Rich syndrome: Review of the literature and analysis of 15 cases. Medicine (Baltimore), 36, 397.

Scadding, J. G. (1960). Chronic diffuse interstitial fibrosis of the lungs. Brit. med. J., $1,443$.

Wilson, M. G. and Mikity, V. G. (1960). A new form of respiratory disease in premature infants. A.M.A. J. Dis. Child., 99, 489. 